\begin{tabular}{|c|l|}
\hline Title & Heritability of male mandible length in the stag beetle Cyclommatus metallifer \\
\hline Author(s) & Gotoh, Hiroki; Fukaya, Keiichi; Miura, Toru \\
\hline Citation & $\begin{array}{l}\text { Entomological Science, 15(4), 430-433 } \\
\text { https://doi.org/40.1111/.1479-8298.2012.00527.x }\end{array}$ \\
\hline Issue Date & 2012-10 \\
\hline Doc URL & http://hdl.handle.net/2115/53417 \\
\hline Rights & The definitive version is available at wileyonlinelibrary.com \\
\hline Type & article (author version) \\
\hline File Information & ES15-4_430-433.pdf \\
\hline
\end{tabular}

Instructions for use 


\section{Heritability of male mandible length in the stag beetle, Cyclommatus metallifer}

Hiroki GOTOH $^{1}$, Keiichi FUKAYA ${ }^{2}$ and Toru MIURA ${ }^{1}$

${ }^{1}$ Laboratory of Ecological Genetics and ${ }^{2}$ Laboratory of Animal Ecology, Graduate School of Environmental Science, Hokkaido University, Sapporo, Japan

Correspondence: Toru Miura, Laboratory of Ecological Genetics, Graduate School of Environmental Science, Hokkaido University, Sapporo, Hokkaido 060-0810, Japan.

Email: miu@ees.hokudai.ac.jp 


\section{Abstract}

Numerous coleopteran species express male-specific "weapon traits" that often show size variations among males, even within a single population. Many empirical studies have demonstrated that environmental conditions during development affect absolute weapon size. However, relatively few studies in horned beetles support the hypothesis that the relationship between weapon size and body size, also referred to as a "scaling relationship" or "static allometry", is largely determined by genetic factors. In this study, the heritability of absolute mandible length and static allometry between mandible length and body size were estimated in the stag beetle Cyclommatus metallifer. While no significant heritable variation was observed in absolute mandible length, high heritability ( $h^{2}$ $=0.57 \pm 0.25)$ was detected in the static allometry between mandible length and body size. This is the first report on the genetic effect on male mandible size in Lucanidae, suggesting that absolute mandible size is largely determined by environmental conditions while the static allometry between weapon size and body size is primarily determined by genetic factors.

Key words: exaggerated morphology, Lucanidae, static allometry, weapon trait. 
The males of numerous coleopteran species exhibit "weapon traits" that are used to compete with rivals for females (Eberhard 1982; Kawano 2006; Emlen 2000, 2008). The size of these weapons typically varies among males, even among those within a single population (Emlen 2000; Kawano 2006; Kodric-Brown et al. 2006). The mandibles of male stag beetles (Coleoptera, Lucanidae) are one of the most prominent examples of such a weapon trait in beetles and their presence has awed scientists and naturalists since the time of Darwin. As with other secondary sexual traits, mandible size varies markedly among males (Hosoya \& Araya 2005; Kawano 2000, 2006; Kodric-Brown et al. 2006). This variation in mandible size is known to be primarily affected by differences in food availability (Gotoh et al. 2011), because the mandible size has been shown to be strongly correlated with body size (Kawano 2000; Kodric-Brown et al. 2006). This relationship between mandible size and body size (i.e. static allometry) is diverse among Lucanidae species (Kawano 2000), and even among populations sharing same body size range (Fujita 2010). One possible explanation of this phenomenon is the existence of genetic effects for determining relative mandible size over body size, probably arising from different selection pressures. However, few experimental studies have been conducted to date investigating the genetic effects of both absolute and relative mandible size in stag beetles. In this study, to determine whether genetic factors affect absolute mandible length and the static allometry between the mandibles and body size, the heritabilities for these two traits were estimated by half-sib analysis in the stag beetle Cyclommatus 
metallifer (Boisduval, 1835). Cyclommatus metallifer is a large, golden-metallic, stag beetle from Indonesia (Fujita 2010). The species is easy to rear and maintain and has been used in a previous study on environmental effects on mandible length (Gotoh et al. 2011). Several specimens belonging to a population of the subspecies C. m. finae (Mizunuma et Nagai, 1991) from Pelen Island in Indonesia (Fujita 2010) were purchased from Hercules-Hercules (Sapporo, Japan). These specimens were used to establish a population that has been maintained for six years in the laboratory. To estimate the heritability of focal trait values, a half-sib experiment was designed for this study (Falconer 1989). Randomly selected males $(n=39)$ were assigned to at least two virgin females (dams). Breeding was facilitated by placing a male and a virgin female together in an $850 \mathrm{ml}$ plastic container for approximately one week. Adults were fed a commercially produced beetle jelly (Za-Okuwa Co., Kawanishi, Japan). After copulation, the females were transferred to another $850 \mathrm{ml}$ plastic container containing approximately $600 \mathrm{ml}$ of decaying wood flakes as a substrate (Quercus spp.; Kuwagata-mat, Hercules-Hercules). After one month, the females were removed (in total, 54 dams successfully laid eggs) and the eggs and larvae were kept in the plastic container under constant darkness at $24 \pm$ $2^{\circ} \mathrm{C}$ until the second instar. Second-instar larvae were kept separately in small plastic containers to determine the exact timing of the second ecdysis, and third instar larvae within 3 days after molting were collected and transferred to a 120 $\mathrm{ml}$ plastic cup filled with decaying wood flakes. These plastic cups were then kept 
under constant darkness at $25 \pm 0.5^{\circ} \mathrm{C}$ until eclosion. As in the parent generation, all of the offspring produced by each female were reared until adult emergence under laboratory conditions, with all of the successfully eclosed sons ( $\mathrm{n}=157$ ) used in the subsequent analysis. Sires and sons were fixed in freezer and then stored for being subjected to morphometric analysis. Prothorax width (indicator of body size) and mandible length (since there is little difference between right and left mandible sizes, only left mandible size was used) were measured to 0.01 mm using a digital slide caliper (Shinwa Rules, Niigata, Japan). Trait values of residual mandible length were defined as follows. First, a two-dimensional scatter plot of the logarithm of body size (X-axis) and the logarithm of mandible length (Y-axis) was constructed using morphometric data from all sires and sons $(n=196)$. Then, a regression line describing the average relationship between mandible length and body size for all of the sampled data was drawn in Excel (Microsoft, Redmond, WA). Residual mandible lengths were then calculated for all males as the difference between actual mandible length and expected mandible length predicted by the fitted line (Fig. 1). Sire-son regressions were conducted to determine narrow-sense heritability $\left(h^{2}\right)$, showing the ratio of additive genetic variance to total phenotypic variance (Falconer 1989). Using this method, statistically significant regressions suggest the existence of heritable components to trait-value variation. Since our data were unbalanced (i.e. number of ovipositing dams per sire and number of sons per dam were different among families), we applied linear mixed models to estimate heritability 
(Visscher et al. 2008). Heritability was estimated by twice the regression coefficient (Falconer 1989). Fitting of the linear-mixed models were performed using the Ime function in the nlme package in R (Pinheiro et al. 2011).

The regression was not significant for absolute mandible length (Fig. $2 ; h^{2}$ $=0.43 \pm 0.25(\mathrm{~S} . \mathrm{E}), \mathrm{P}=0.0964)$, suggesting that sire mandible length had little effect on the mandible length of its sons. Considering the marked effect of nutrition on weapon size reported previously in this and other coleopteran species (Emlen 1994, Moczek 1998, Karino et al. 2004, Gotoh et al. 2011), this result could primarily be attributed to environmental effects rather than to a low additive genetic variance. Conversely, the regression was statistically significant for residual mandible length (Fig. $3 ; h^{2} \pm$ S.E. $=0.57 \pm 0.25, P=0.0277$ ). High estimate value of the regression slope indicates that the static allometry between body and mandible size possesses a large heritable genetic variance. It is thus possible that the diversity in static allometry observed within the Lucanidae is attributed to genetic differences among species or populations rather than differences in environmental conditions.

Generally in beetles with weapons, the weapon function in combat with rivals is largely dependent upon relative sizes between weapon and body rather than absolute sizes (Eberhard 1982; Moczek et al. 2002; Hongo 2003). In addition, since possessing weapons could be costs (Emlen 2001), appropriate resource allocation to weapons might be critical for their fitness (Moczek 2003; Pomfret \& Knell 2008). Ecological factors such as habitats, fighting behavior and 
population density which may affect the efficiency and cost of possessing weapon, is suggested to act as selective pressures that are different among the species or populations. In fact, using horned beetles (Scarabaeidae), Moczek et al. (2002) demonstrated that the distinct static allometry of horns over body size evolved rapidly in an Australian introduced population, while Emlen (1996) showed that artificial selection for residual horn length over several generations was sufficient to alter the static allometry. Considering these previous studies and our results, static allometry between weapon and body size in Cyclommatus metallifer (and possibly various static allometry patterns in other Lucanidae species) may be deeply rooted in their behavior and/or ecology, which means that the observed static allometry pattern likely reflect the selection pressures acting on male weapons.

\section{ACKNOWLEDGMENTS}

We would like to thank our colleagues and Mr. F. Nomano for their advice and critical comments on this study. This work was supported by a Grant-in-Aid for Young Scientists (No. 21677001) from the Ministry of Education, Culture, Sports, Science and Technology of Japan. HG was supported by JSPS Research Fellowships for Young Scientists. 


\section{REFERENCES}

Eberhard WG (1982) Beetle horn dimorphism: Making the best of a bad lot. The American Naturalist 119, 420-426.

Emlen DJ (1994) Environmental control of horn length dimorphism in the beetle Onthophagus acuminatus (Coleoptera: Scarabaeidae). Proceedings of the Royal Society B 256, 131-136.

Emlen DJ (1996) Artificial selection on horn length-body size allometry in the horned beetle Onthophagus acuminatus (Coleoptera: Scarabaeidae). Evolution 50, 1219-1230.

Emlen DJ (2000) Integrating development with evolution: a case study with beetle horns. Bioscience 50, 403-418.

Emlen DJ (2001) Costs and the diversification of exaggerated animal structures. Science 291, 1534-1536.

Emlen DJ (2008) The evolution of animal weapons. Annual Review of Ecology, Evolution and Systematics 39, 387-413.

Falconer DS (1989) Introduction to quantitative genetics. John Wiley and Sons, New York.

Fujita H. (2010) The Lucanid beetles of the world. Mushi-sha. (In Japanese).

Gotoh H, Cornette R, Koshikawa S, Okada Y, Lavine LC, Emlen DJ, Miura T. (2011) Juvenile hormone regulates extreme mandible growth in male stag beetles. PLOS ONE 6 (6): e21139. doi:10.1371/journal.pone.0021139. 
Hongo Y (2003) Appraising behaviour during male-male interaction in the Japanese horned beetle Trypoxylus dichotomus septentrionalis Kono. Behaviour 140, 501-517.

Hosoya T, Araya K (2005) Phylogeny of Japanese stag beetles (Coleoptera: Lucanidae) inferred from 16S mtrRNA gene sequences, with reference to the evolution of sexual dimorphism of mandibles. Zoological Science 22, 1305-1318.

Karino K, Seki N, Chiba M (2004) Larval nutritional environment determines adult size in Japanese horned beetles Allomyrina dichotoma. Ecological Research 19, 663-668.

Kawano K (2000) Genera and allometry in the stag beetle family Lucanidae, Coleoptera. Annals of the Entomological Society of America 93, 198-207.

Kawano K (2006) Sexual dimorphism and the making of oversized male characters in Beetles (Coleoptera). Annals of the Entomological Society of America 99, 327-341.

Kodric-Brown AK, Sibly RM, Brown JH (2006) The allometry of ornaments and weapons. Proceedings of the National Academy of Sciences of the USA 103, 8733-8738.

Moczek AP (1998) Horn polyphenism in the beetle Onthophagus taurus: larval diet quality and plasticity in parental investment determine adult body size and male horn morphology. Behavioral Ecology 9, 636-641. 
Moczek AP, Hunt J, Emlen DJ, Simmons LW (2002) Threshold evolution in exotic populations of a polyphenic beetle. Evolutionary Ecology Research 4, 587-601.

Moczek AP (2003) The behavioral ecology of threshold evolution in a polyphenic beetle. Behavioral Ecology 14, 841-854.

Pinheiro J, Bates D, DebRoy S, Sarkar D, the R Development Core Team (2011). nlme: Linear and Nonlinear Mixed Effects Models. R package version 3.1-102.

Pomfret JC, Knell RJ (2008) Crowding, sex ratio and horn evolution in a South African beetle community. Proceedings of the Royal Society B 275, 315-321. Visscher PM, Hill WG, Wray NR (2008) Heritability in the genomics era - concepts and misconceptions. Nature Reviews Genetics 9, 255-266. 


\section{FIGURE LEGENDS}

Figure 1 Schematic explanation of the method used to calculate the trait value of static allometry between mandible length and body size. $\mathrm{X}$ and $\mathrm{Y}$ axes indicate logarithmic values of prothorax width (indicator of body-size) and mandible length, respectively. Open circles represent individuals used in this study ( $\mathrm{n}=$ 196), and the solid line is a regression line based on all plots $(y=2.749 x-3.350$, $\left.\mathrm{R}^{2}=0.719\right)$. The regression line describes an average static allometry between the two characters. Residual mandible lengths were calculated for all males as the difference between actual mandible length and the expected mandible length inferred from the regression line. The dashed line represents the residual mandible length of a hypothetical individual (closed circle). LN: Natural logarithm

Figure 2 Relationship between absolute mandible lengths in sires $(n=39)$ and means of sons for each full-sib family $(n=54) . X$ and $Y$ axes indicate mandible lengths of sires and means of sons for each full-sib family, respectively. The regression between the two values was not significant $\left(h^{2} \pm\right.$ S.E. $=0.43 \pm 0.25$, $P=0.0964)$

Figure 3 Relationship between residual mandible lengths of sires $(n=39)$ and means of sons for each full-sib family $(n=54) . X$ and $Y$ axes indicate residual mandible lengths of sires and means of sons for each full-sib family, respectively. 
The regression for the residual mandible length was significant $\left(h^{2} \pm\right.$ S.E. $=0.57$ $\pm 0.25, P=0.0277)$. 


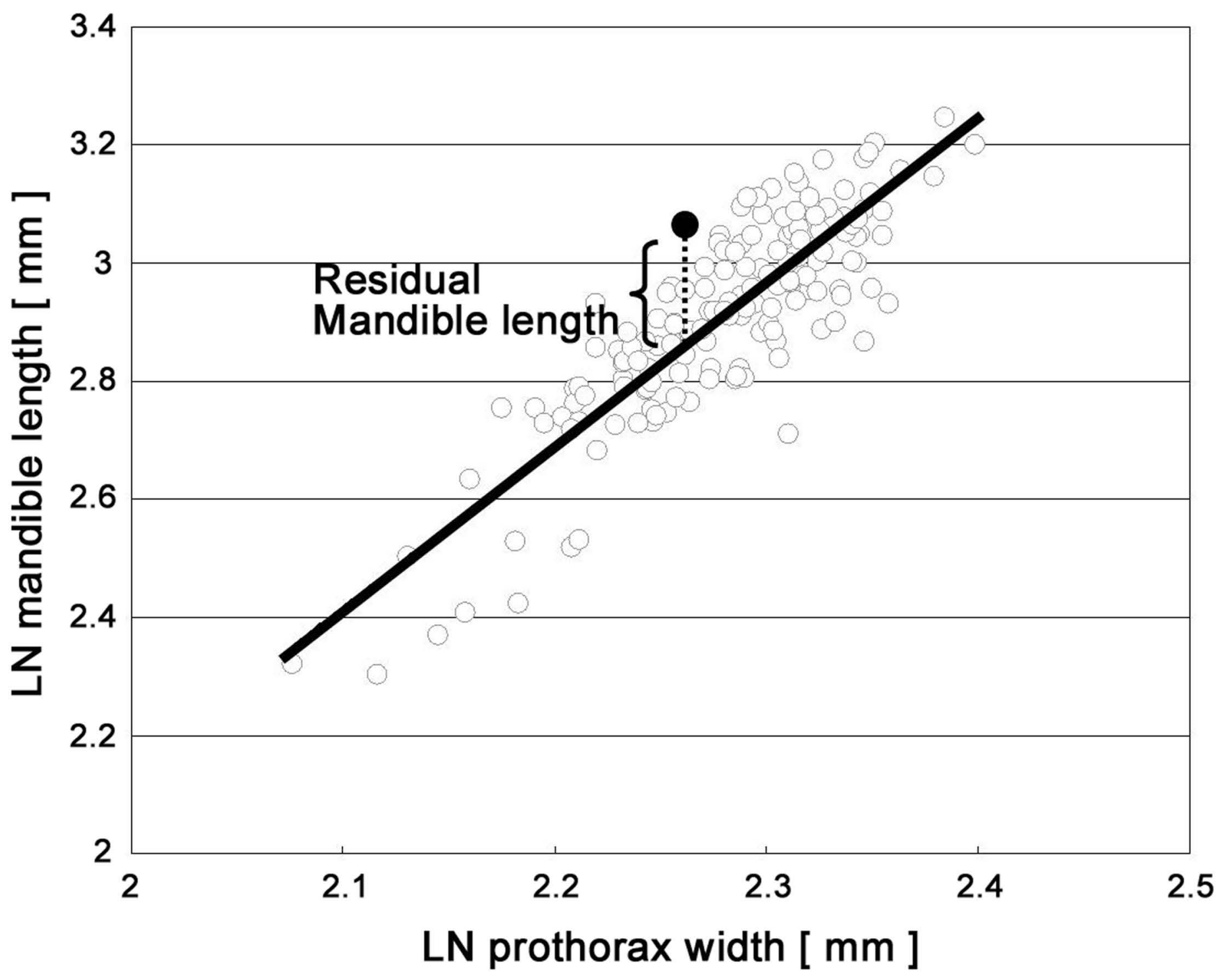




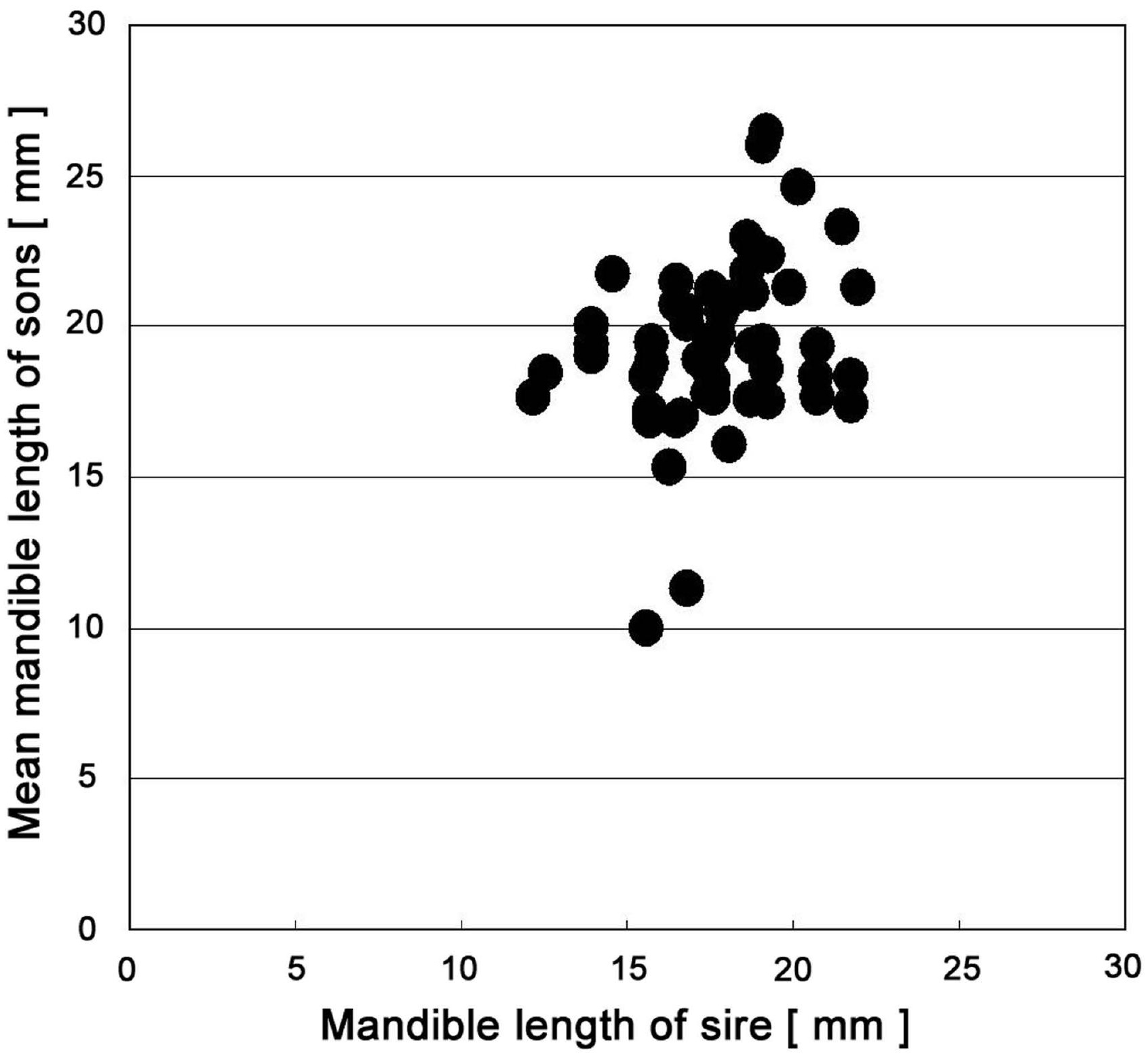


